\title{
Diet and lifestyle behaviour disruption related to the pandemic was varied and bidirectional among US and UK adults participating in the ZOE COVID Study
}

\author{
Mohsen Mazidi1,2,15, Emily R. Leeming ${ }^{1,15}$, Jordi Merino3,4,5, Long H. Nguyen ${ }^{6,7}$, Somesh Selvachandran ${ }^{8}$, \\ Joan Capdavila Pujal ${ }^{8}$, Tyler Maher ${ }^{2}$, Kirstin Kadé ${ }^{8}$, Benjamin Murray ${ }^{9}$, Mark S. Graham (1) \\ Carole H. Sudre ${ }^{9,10,11}$, Jonathan Wolf $\mathbb{1}^{8}{ }^{8}$, Christina Hu ${ }^{8}$, David A. Drew ${ }^{6,7}$, Claire J. Steves ${ }^{1}$, \\ Sebastien Ourselin ${ }^{9}$, Christopher Gardner ${ }^{12}$, Tim D. Spector ${ }^{1,8,16}$, Andrew T. Chan ${ }^{4,6,7,13,16}$, \\ Paul W. Franks ${ }^{13,14,16}$, Rachel Gibson ${ }^{2,16}$ and Sarah E. Berry ${ }^{2,16} \bowtie$
}

Evidence of the impact of the COVID-19 pandemic on health behaviours in the general population is limited. In this retrospective longitudinal study including UK and US participants, we collected diet and lifestyle data pre-pandemic $(896,286)$ and peri-pandemic $(291,871)$ using a mobile health app, and we computed a bidirectional health behaviour disruption index. Disruption of health behaviour was higher in younger, female and socio-economically deprived participants. Loss in body weight was greater in highly disrupted individuals than in those with low disruption. There were large inter-individual changes observed in $\mathbf{4 6}$ health and diet behaviours measured peri-pandemic compared with pre-pandemic, but no mean change in the total population. Individuals most adherent to less healthy pre-pandemic health behaviours improved their diet quality and weight compared with those reporting healthier pre-pandemic behaviours, irrespective of relative deprivation; therefore, for a proportion of the population, the pandemic may have provided an impetus to improve health behaviours. Public policies to tackle health inequalities widened by the pandemic should continue to prioritize diet and physical activity for all, as well as more targeted approaches to support younger females and those living in economically deprived areas.

M andatory public health initiatives to control and limit the spread of coronavirus disease 2019 (COVID-19) have led to dramatic changes in day-to-day routines, resulting in increased social isolation ${ }^{1,2}$, employment and financial insecurities ${ }^{3}$, and an altered food environment ${ }^{4}$. This places most of the world's population in a unique global experiment on a massive scale. Small European-based studies have observed the exacerbation of unfavourable diet and lifestyle behaviours attributable to these changes, such as increased sedentary behaviour, more snacking, less fresh food consumption and weight gain, although the published data are inconclusive ${ }^{5-7}$.

At an individual level, major life events are associated with changes in health behaviours such as alcohol intake ${ }^{8}$, sleep ${ }^{9,10}$, diet ${ }^{11,12}$ and physical activity ${ }^{13}$. The complex interrelation of these health behaviours potentially mediates increases in body weight observed during adulthood ${ }^{14-16}$, impacting the number of adults living with excess weight and consequent morbidities ${ }^{17-20}$, which is a significant public health threat ${ }^{21}$. Understanding how health behaviours change in the context of a prolonged pandemic is therefore critical to improve our understanding of its long-term consequences.

In a retrospective longitudinal observational cohort study of 896,286 participants from the ZOE COVID Study, we describe the self-reported impact of the COVID-19 pandemic on diet and health behaviours using a composite disruption index (DI). We determine the association of diet and lifestyle behaviours with weight change during the pandemic, and we explore the relationship between pre-pandemic behavioural patterns and changes in specific diet and lifestyle behaviours.

There were large inter-individual changes observed in all of the 46 health and diet behaviours measured peri-pandemic versus pre-pandemic, but no mean change was observed in the total population. Individuals most adherent to less healthy pre-pandemic diet and health behaviours were more inclined to improve their overall diet quality and lose weight, irrespective of relative deprivation,

'Department of Twin Research, King's College London, London, UK. ²Department of Nutritional Sciences, King's College London, London, UK. ${ }^{3}$ Diabetes Unit and Center for Genomic Medicine, Massachusetts General Hospital, Boston, MA, USA. ${ }^{4}$ Department of Medicine, Harvard Medical School, Boston, MA, USA. ${ }^{5}$ Programs in Metabolism and Medical \& Population Genetics, Broad Institute, Cambridge, MA, USA. ${ }^{6} \mathrm{Clinical}$ and Translational Epidemiology Unit, Massachusetts General Hospital and Harvard Medical School, Boston, MA, USA. 7 Division of Gastroenterology, Massachusetts General Hospital and Harvard Medical School, Boston, MA, USA. ${ }^{8}$ ZOE Global Ltd, London, UK. ${ }^{9}$ School of Biomedical Engineering and Imaging Sciences, King's College London, London, UK. ${ }^{10} \mathrm{MRC}$ Unit for Lifelong Health and Ageing at UCL, University College London, London, UK. "Centre for Medical Image Computing, University College London, London, UK. ${ }^{2}$ Stanford Prevention Research Center, Department of Medicine, Stanford University, Stanford, CA, USA. ${ }^{13}$ Harvard T.H. Chan School of Public Health, Harvard University, Boston, MA, USA. ${ }^{14}$ Lund University Diabetes Center, Department of Clinical Sciences, Lund University, Malmö, Sweden. ${ }^{15}$ These authors contributed equally: Mohsen Mazidi, Emily R. Leeming. ${ }^{16}$ These authors jointly supervised this work: Tim D. Spector, Andrew T. Chan, Paul W. Franks, Rachel Gibson, Sarah E. Berry. $\varpi_{e}$-mail: sarah.e.berry@kcl.ac.uk 
while those most adherent to healthier pre-pandemic behaviours exhibited little change in health behaviours. Disruption of health behaviours resulting from the pandemic was higher in the younger, female and socio-economically deprived segments of the population. However, greater disruption was associated with more variable change in weight and greater weight loss compared with less disrupted individuals, suggesting that the disruption resulted in more favourable diet and lifestyle changes for some people. Structural equation modelling revealed that changes in physical activity and diet quality during the pandemic were the most relevant factors associated with weight change.

Our data provide quantitative evidence about the impact of the COVID-19 pandemic on lifestyle behaviours and indicate that the perceived negative impact of the pandemic on health behaviours, and factors that exacerbate this, may not hold true for all people ${ }^{22}$. Indeed, the pandemic may have provided the impetus to improve many diet and lifestyle behaviours among a large proportion of the population.

\section{Results}

Within the ZOE COVID Study, we launched a diet and lifestyle retrospective longitudinal observational cohort study (from 31 July to 25 September 2020 in the United Kingdom and from 25 September to 30 November 2020 in the United States). The participants were invited to complete a retrospective questionnaire (Methods) assessing diet quality, diet habits and lifestyle behaviours at two time points: (1) peri-pandemic (the previous month before participant access) and (2) pre-pandemic (the month of February 2020). Baseline participant characteristics including demographics and comorbidities were also assessed from the ZOE COVID Study ${ }^{23}$. For the UK data, the index of multiple deprivation (IMD), considered a surrogate measure of deprivation across several domains (including income, employment and education) by geographical region ${ }^{24}$, was assigned to the participants.

Characteristics of the 896,286 survey respondents. After the exclusion of replicates, individuals $<18$ years old, pregnant women, non-UK and non-US participants and implausible anthropometric measures (Methods), data were available for $n=896,286$ peri-pandemic and $n=291,871$ pre- and peri-pandemic participants.

The characteristics of the participants who completed the diet and lifestyle survey peri-pandemic are summarized in Table 1 and Supplementary Tables 1 and 2. Compared with the average UK population (Supplementary Table 1), the UK participants who completed the survey were older (mean age 52 versus 41 years), had a lower body mass index (BMI) (25 versus $27 \mathrm{~kg} \mathrm{~m}^{-2}$ ), resided in less deprived areas (for IMD range $8-10,40 \%$ versus $30 \%$; for IMD range $1-3,10 \%$ versus $30 \%$ ) and were less likely to smoke. The proportions of males and non-white UK ethnicities were also lower among the surveyed UK participants than in the general population (33\% versus $49 \%$ and $4 \%$ versus $14 \%$, respectively). The proportions of respondents across geographical regions in the United Kingdom were broadly similar to the UK population distribution. Similar patterns were observed for the US cohort (Table 1 and Supplementary Table 1).

Prior to data analysis, the UK cohort was divided into discovery $(n=380,847)$ and validation $(n=448,321)$ sets, owing to slight differences in data collection processes (Methods and Supplementary Table 2). Data from 67,118 participants from the United States were used as an independent replication cohort (Methods).

Application of a lifestyle DI. To quantify lifestyle disruption attributable to the pandemic, we computed a DI including five metrics: diet quality score (DQS) ${ }^{25}$, alcohol frequency, physical activity, snacking frequency (food consumed outside of main meals) and weekday sleep duration (Methods). These domains were selected to capture the primary diet and lifestyle behaviours associated with multiple health outcomes, including obesity ${ }^{26-31}$. The DI was independent of the direction of change, ranged from 0 (no disruption) to 5 (change in all five domains) and approximated a normal distribution (Fig. 1a). In the UK discovery cohort ( $n=201,301$; pre- and peri-pandemic data), most participants experienced a moderate level of disruption $(65 \%$; DI $\geq 2$ ), while $15 \%$ had a high level of disruption (DI $\geq 4)$. The DI (Fig. 1a) differed (chi-squared test) according to sex $(P<0.001$; effect size, 0.122$)$, age category $(P<0.001$; effect size, 0.268), assigned level of deprivation $(P<0.001$; effect size, 0.050) and geographical location $(P<0.001$; effect size, 0.014 ), with greater DIs among younger individuals, females and participants living in more deprived areas. Similar patterns were observed for the US replication cohort ( $n=14,473$; pre- and peri-pandemic data). Participant characteristics according to the DI scores for the United Kingdom and United States are detailed in Supplementary Table 3.

For body weight change (in the UK discovery cohort $(n=201,301$; pre- and peri- pandemic data)), only a small mean change (mean (10th percentile, 90th percentile)) of $-0.2(-4.4,3.6) \mathrm{kg}$ was observed. However, body weight change was highly variable among individuals, with $33 \%$ of participants losing a mean of $4.4(-8.6$, $-0.9) \mathrm{kg}$ and $34 \%$ gaining a mean of $3.7(0.9,6.4) \mathrm{kg}$ during the pandemic (Supplementary Table 4). A similar pattern and magnitude of change was also observed for the UK validation and US replication cohorts (Supplementary Table 4).

A greater disruption was associated with a larger and more variable bidirectional change in weight (both loss and gain) in both the UK and US cohorts (Fig. 1b and Supplementary Table 3). Weight loss and gain in the high DI group (mean (10th percentile, 90th percentile)) was $-5.5(-11.0,-1.0) \mathrm{kg}$ and $4.2(1.0,8.0) \mathrm{kg}$, respectively, compared with $-3.5(-6.4,-0.9) \mathrm{kg}$ and $3.3(0.5,5.4) \mathrm{kg}$, respectively, in the low DI group (all $P<0.001$ ).

After adjusting for potential confounders (age and sex), the pattern of association between the DI and weight changes was similar but stronger among individuals living in areas with low deprivation (IMD, $8-10 ; \beta=-0.035 ; 95 \%$ confidence interval, $-0.217,-0.145$ ) compared with high deprivation (IMD, $1-3 ; \beta=-0.021 ; 95 \%$ confidence interval, $-0.153,-0.058)$. When stratified according to DI group and deprivation index, there was a similar magnitude of weight loss between levels of deprivation, and only a moderately higher weight gain in the UK group residing in more deprived areas $(4.4 \mathrm{~kg})$ versus the group in less deprived areas $(4.0 \mathrm{~kg})$ within the highly disrupted group (Supplementary Table 3 ). This observation suggests that community-level deprivation factors may not impair the potential positive behavioural effects of disruption but may exacerbate the negative effects to a small extent, with individuals living in more deprived areas being more susceptible to weight gain.

The association of changes in the five lifestyle behaviour domains captured by the DI and age ${ }^{32}$ with body weight change (bidirectional) was examined using structural equation modelling (Fig. $2 \mathrm{a}, \mathrm{d})$. For individuals who gained weight $(n=68,607)$, reductions in physical activity, diet quality and sleep and an increase in snacking were moderately associated with weight gain. Among individuals who lost weight $(n=65,327)$, contrasting associations were observed with an additional moderate association of reduced alcohol frequency with weight loss. This trend remained when examined within strata of community-level deprivation, except for sleep, which was not associated with weight gain or loss in those living in more deprived areas (Supplementary Table 5 and Fig. 2b,c,e,f). In accordance with most population-based strategies, our results show that diet quality and physical activity can be promising targets to prevent weight gain across all socio-economic classes during a global pandemic.

To determine any differential effect of pre-pandemic weight on the relationship between the DI components and weight change, 
Table 1 | Characteristics of app users

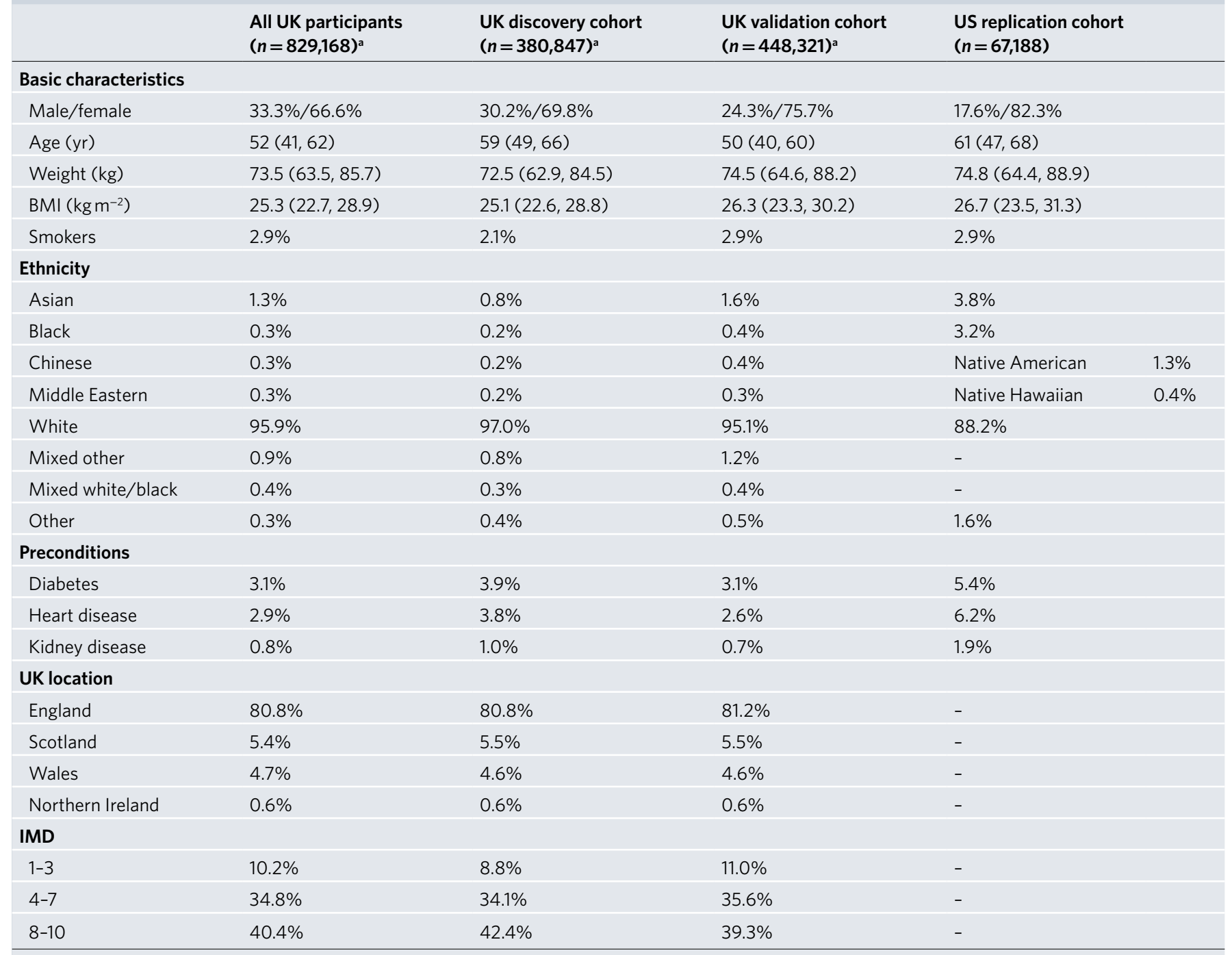

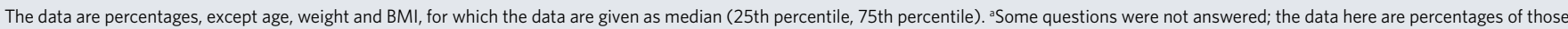
that answered. Those who completed the peri-pandemic survey only are not included in this table. The full dataset can be found in Supplementary Table 1 .

we also stratified according to normal weight $\left(\mathrm{BMI}<25 \mathrm{~kg} \mathrm{~m}^{-2}\right.$; $n=97,915)$ and overweight/obese (BMI $\left.\geq 25 \mathrm{~kg} \mathrm{~m}^{-2} ; n=103,386\right)$ (Supplementary Table 4). Reductions in physical activity and DQS and an increase in snacking were most closely associated with weight gain in both groups, with a stronger association in the overweight/obese individuals $(\beta=-0.055,-0.044$ and 0.05 , respectively, all $P<0.05)$ versus normal-weight individuals $(\beta=-0.014,-0.019$ and 0.028 , respectively, all $P<0.05$ ).

Individual-level changes in diet and lifestyle behaviours. There was minimal population average change in all diet and lifestyle variables in response to the pandemic (in the UK and US cohorts) but large individual-level changes (Table 2, Fig. 3a-c and Supplementary Tables 4,6 and 7). For example, in the UK discovery cohort $(n=201,301)$, despite minimal mean change in the DQS (mean (10th percentile, 90th percentile), $0.2(-2.0,2.0))$, more participants increased their score $(37 \%$ by a mean of $1.7(1.0,3.0))$ than decreased it $(26 \%$ by a mean of $-1.6(-3.0,-1.0)$ ). Likewise, a higher proportion of participants $(31.0 \%)$ increased their daily fruit and vegetable portions (by mean $1.85(1.0,3.0)$, from $4.3(0.2,7.0)$ to $6.1(3.5,9.0)$ portions per day) than decreased them $(22.0 \%)$ (by mean $-1.68(-3.0,-1.0)$, from $6.04(3.0,9.0)$ to $4.35(2.0,7.0)$ portions per day), consistent with a level that would confer significant health effects ${ }^{27,33}$. There was only a minimal mean change in the number of snacks consumed per day (by mean $-0.1(-1.0,1.0)$ ), but a large number of individuals increased their intake (16\%; by mean 1.4 snacks per day (1.0, 2.0) (from 1.1 per day)) and decreased their intake (23\%; by mean -1.4 snacks per day $(-2.0,-1.0)$ (from 2.6 per day)). More people increased their weekday sleep (15\%) than decreased it (9\%), although the magnitude of change was similar (mean $\pm 1.2 \mathrm{~h}$ ). Interestingly, for frequency of alcohol intake, the proportion of people increasing (18.2\%) was greater than the proportion decreasing (11.2\%), while the inverse was the case for the amount consumed at each drinking event (10.6\% increasing versus $13.4 \%$ decreasing units), suggesting that the total amount of alcohol consumption (frequency $\times$ units) may have been unchanged. Notably, most participants reported no change $(92.8 \%)$ in food access, probably a reflection of the survey demographic, although more participants reported a decrease in food access (5.8\%) than an increase (1.4\%).

A similar pattern of change in the individual variables was observed in the UK validation cohort and US replication cohort except for physical activity levels. In the United Kingdom, the proportions of participants increasing and decreasing their physical activity were similar (in both cohorts), yet in the United States, the 


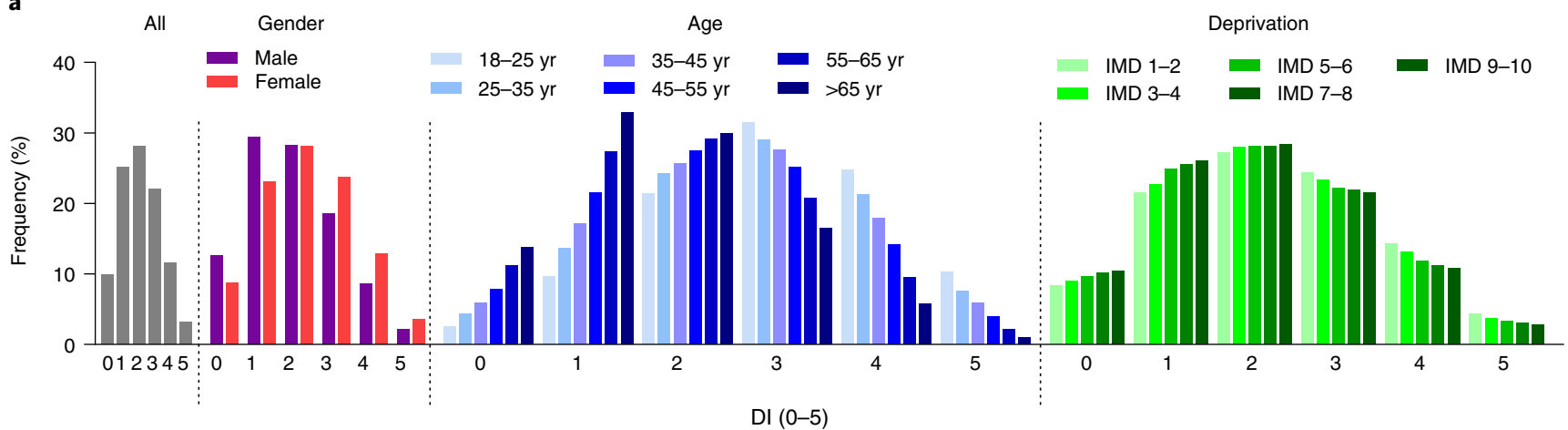

b
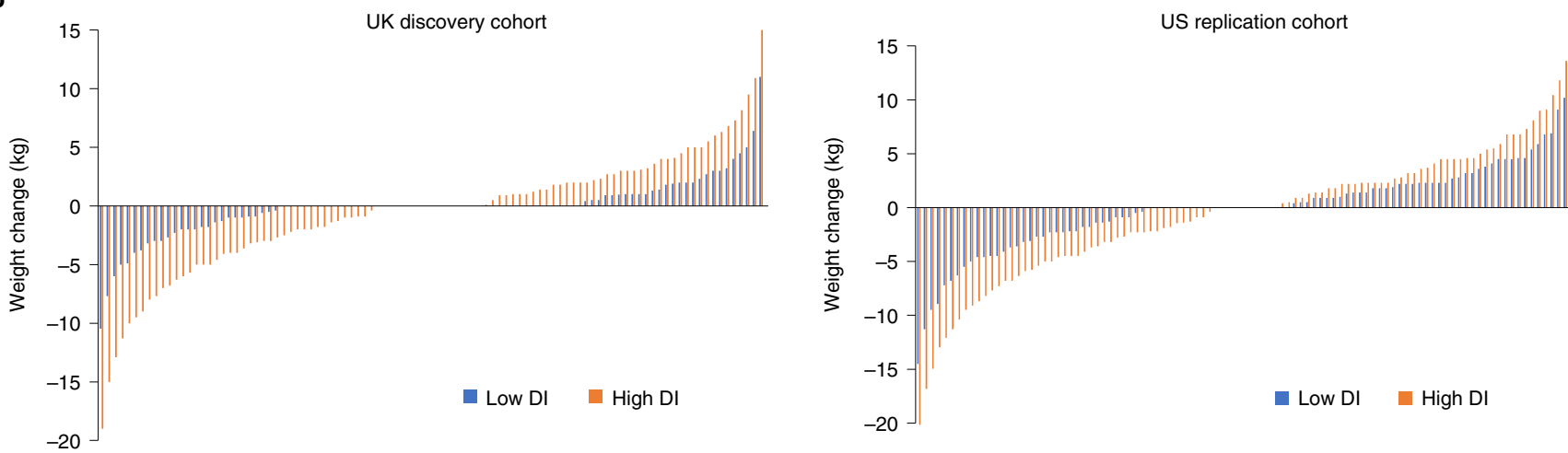

c

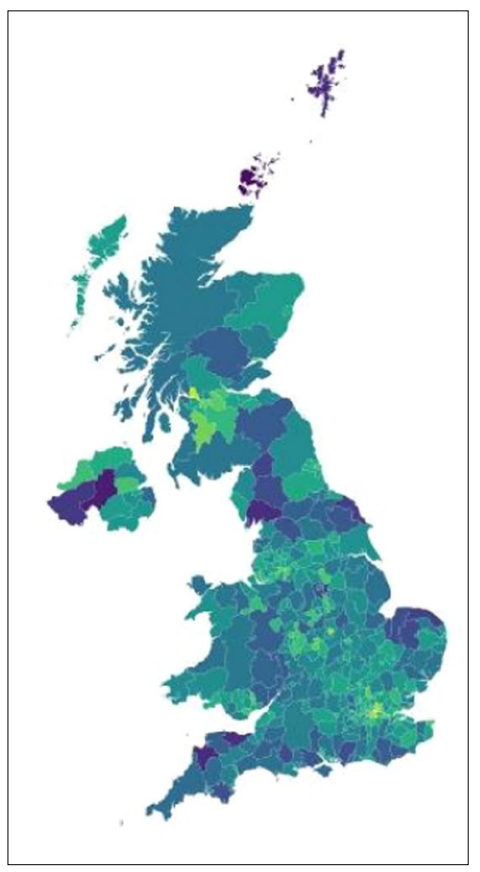

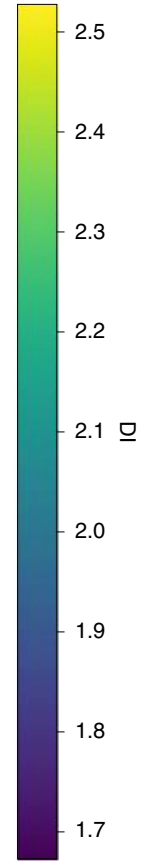

d

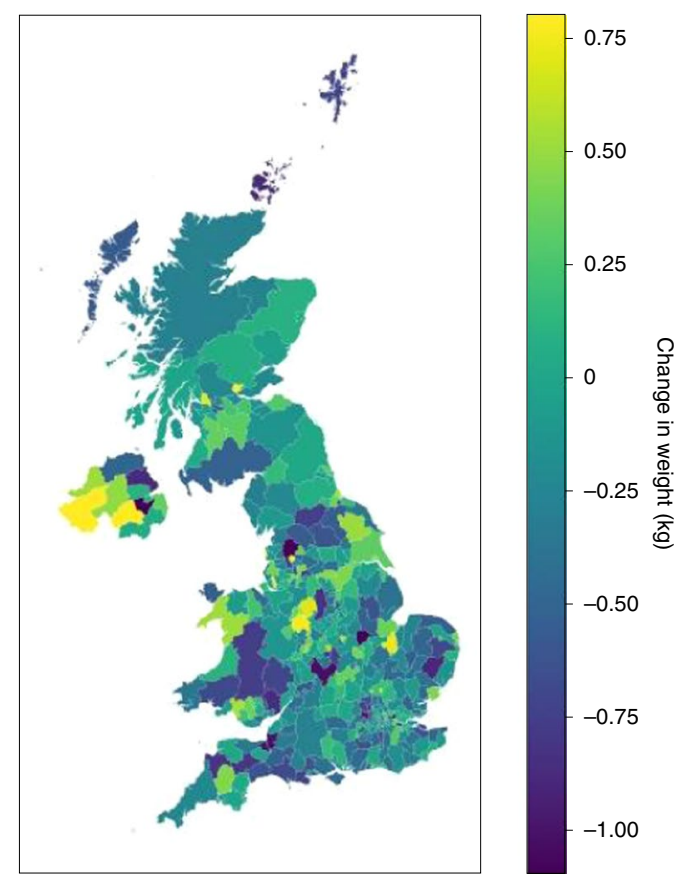

Fig. 1 | DI population distribution and weight change. a, Community-level DI on a scale of 0 to 5 including five domains (DQS, snacking frequency, alcohol intake frequency, physical activity and weekday sleep duration), stratified according to sex, age and IMD based on residence in the UK cohort $(n=201,301)$. b. Change in body weight during the pandemic (pre- minus peri-pandemic values) stratified according to low $(\leq 1)$ or high $(\geq 4) \mathrm{DI}$ in the UK discovery $(n=201,301)$ and US replication cohorts $(n=14,473)$, coefficient of variation for those with high DI (UK discovery cohort, $2,130 \%$; US replication cohort, 2,288\%) and coefficient of variation for those with a low DI (UK discovery cohort, 1,488\%; US replication cohort, 970\%). c,d, Geographical distribution of DI (c) and change in weight (d) in the UK discovery cohort $(n=201,301)$. The data are provided in Supplementary Table 3.

proportion of participants decreasing their physical activity (35.4\%) was greater than those increasing it (24.6\%) (Table 2 and Fig. 3 ). The moderately greater magnitude of change in the UK validation and
US replication cohorts than in the UK discovery cohort is probably a consequence of the different data collection methods (Methods). All other variables from the questionnaire, including full Leeds 


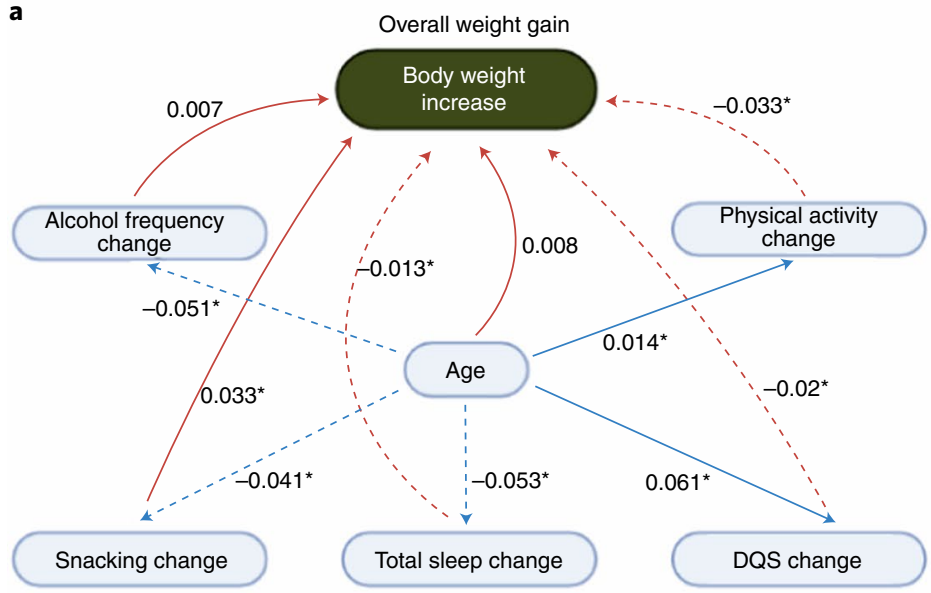

b

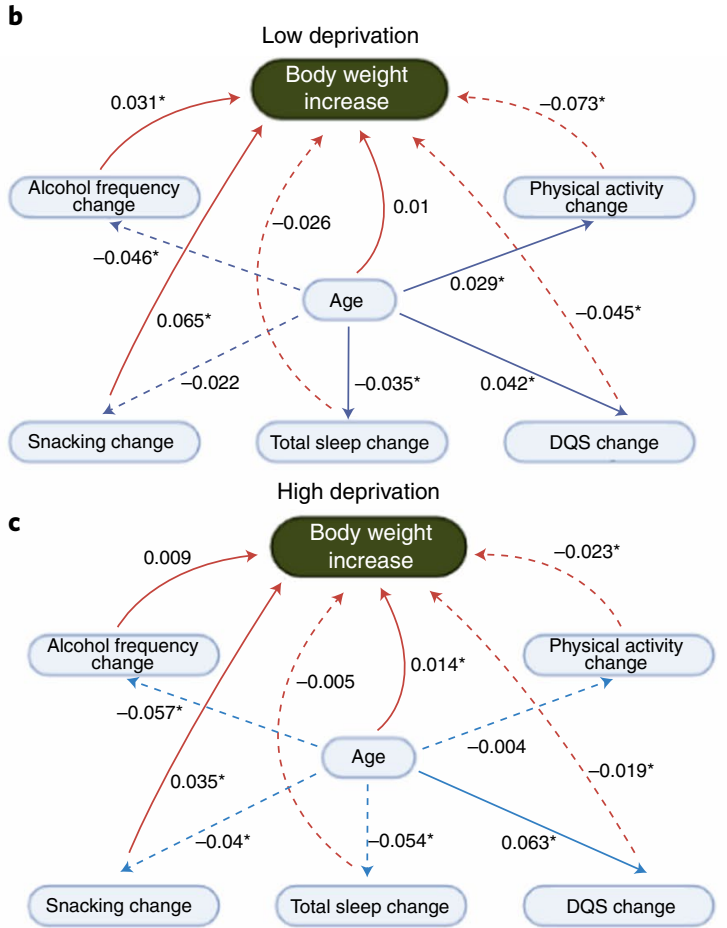

d
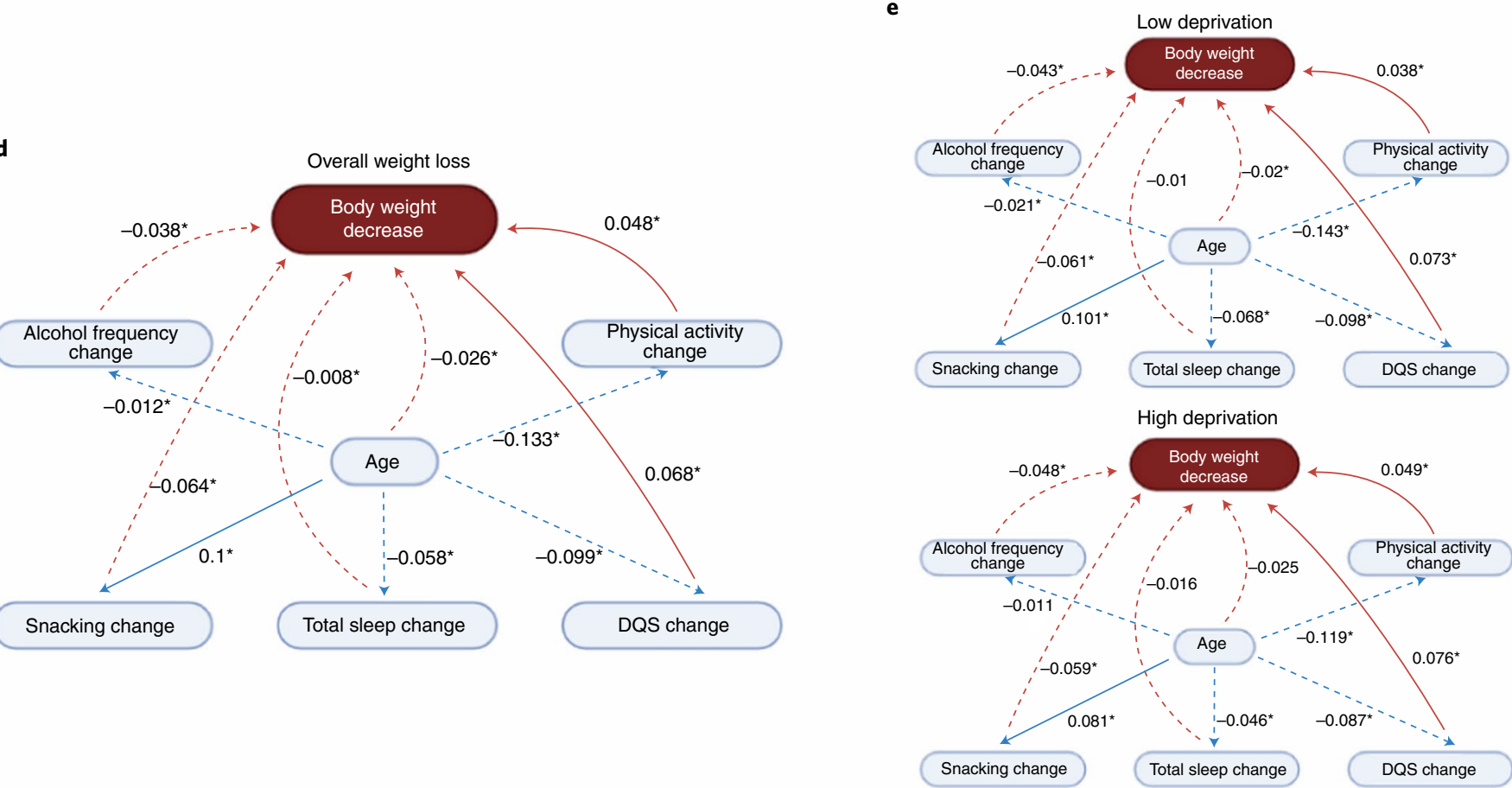

Fig. 2 Association between diet and lifestyle and change in weight pre-pandemic to peri-pandemic. a-f, Structural equation modelling showing the associations between exposures identified by machine learning and outcome (body weight increase/decrease): overall increase $(n=68,607)$ (a), body weight increase in the subpopulation in low deprivation areas (IMD 8-10; $n=28,054)(\mathbf{b})$, body weight increase in the subpopulation in high deprivation areas (IMD $1-3 ; n=6,779)$ (c), overall decreased body weight $(n=65,327)$ (d), body weight decrease in the subpopulation in low deprivation areas (IMD 8-10; $n=28,015)$ (e) and body weight decrease in the subpopulation in high deprivation areas (IMD 1-3;n=5,533) (f). The blue arrows represent indirect effects on body weight. The red arrows represent direct effects on body weight. The dashed arrows represent inverse effects on body weight. The numbers represent the strength of association (standardized $\beta$ coefficients). ${ }^{\star} P<0.05$. The data are provided in Supplementary Table 5. Figure created with BioRender.com.

Short Form Food Frequency Questionnaire (LSF-FFQ) data, are reported for pre- and peri-pandemic (as well as the change) in Supplementary Tables 4, 6 and 7.
Association between pre-pandemic diet and lifestyle and peri-pandemic change. To explore the impact of pre-pandemic diet and lifestyle behaviours on the variable response to the 
Table 2 | Pre- and peri-pandemic data

\begin{tabular}{|c|c|c|c|c|c|c|c|}
\hline & \multicolumn{3}{|c|}{ Averaged data } & \multicolumn{2}{|c|}{ Increase } & \multicolumn{2}{|c|}{ Decrease } \\
\hline & Pre-pandemic & Peri-pandemic & $\begin{array}{l}\text { Change } \\
\text { (10th, 90th) }\end{array}$ & $\begin{array}{l}\text { Participants } \\
\text { (\%) }\end{array}$ & $\begin{array}{l}\text { Change } \\
\text { (10th, 90th) }\end{array}$ & $\begin{array}{l}\text { Participants } \\
\text { (\%) }\end{array}$ & $\begin{array}{l}\text { Change } \\
\text { (10th, 90th) }\end{array}$ \\
\hline \multicolumn{8}{|l|}{ Anthropometrics } \\
\hline Weight (kg) & $75.6(18.0)$ & $75.5(17.7)$ & $-0.2(-4.4,3.6)$ & 34.0 & $3.7(0.9,6.4)$ & 33.0 & $-4.4(-8.6,-0.9)$ \\
\hline $\mathrm{BMI}\left(\mathrm{kg} \mathrm{m}^{-2}\right)$ & $26.4(5.7)$ & $26.3(5.6)$ & $-0.1(-1.5,1.3)$ & 34.0 & $1.3(0.3,2.4)$ & 33.0 & $-1.5(-3.0,-0.3)$ \\
\hline Fruit portions & $2.2(1.6)$ & $2.3(1.7)$ & $0.1(-1.0,1.0)$ & 21.0 & $1.6(1.0,2.0)$ & 15.0 & $-1.5(-2.0,-1.0)$ \\
\hline Vegetable portions & $2.8(1.4)$ & $2.8(1.5)$ & $0.1(-1.0,1.0)$ & 19.0 & $1.4(1.0,2.0)$ & 16.0 & $-1.3(-2.0,-1.0)$ \\
\hline Glasses of juice & $0.5(1.4)$ & $0.5(1.5)$ & $0.0(0.0,0.0)$ & 7.0 & $2.8(0.7,10.0)$ & 7.0 & $-2.5(-6.0,-1.0)$ \\
\hline Weekday sleep (h) & $7.0(1.0)$ & $7.1(0.9)$ & $0.1(0.0,1.0)$ & 15.0 & $1.2(1.0,2.0)$ & 9.0 & $-1.2(-2.0,-1.0)$ \\
\hline Weekend sleep (h) & $7.4(1.1)$ & $7.3(1.0)$ & $0.0(-1.0,0.0)$ & 8.0 & $1.2(1.0,2.0)$ & 11.0 & $-1.2(-2.0,-1.0)$ \\
\hline Eats breakfast (\%) & 83.3 & 84.1 & & - & - & - & - \\
\hline Worried about control (\%) & 15.6 & 18.4 & & - & - & - & - \\
\hline \multicolumn{8}{|l|}{ UK validation cohort } \\
\hline \multicolumn{8}{|l|}{ Anthropometrics } \\
\hline Weight (kg) & $78.3(19.2)$ & $78.3(18.7)$ & $0.1(-6.4,5.9)$ & 49.0 & $4.3(1.0,8.0)$ & 36.0 & $-5.7(-11.0,-1.0)$ \\
\hline BMI $\left(\mathrm{kg} \mathrm{m}^{-2}\right)$ & $27.5(6.2)$ & $27.5(6.1)$ & $0.1(-2.3,2.0)$ & 49.0 & $1.5(0.4,2.8)$ & 36.0 & $-2.0(-3.8,-0.4)$ \\
\hline \multicolumn{8}{|l|}{ LSF-FFQ diet questionnaire } \\
\hline DQS & $10.6(1.9)$ & $10.9(1.9)$ & $0.4(-2.0,3.0)$ & 42.0 & $2.1(1.0,4.0)$ & 31.0 & $-1.9(-3.0,-1.0)$ \\
\hline Fruit portions & $2.0(1.5)$ & $2.1(1.6)$ & $0.1(-1.0,1.0)$ & 27.0 & $1.6(1.0,2.0)$ & 23.0 & $-1.5(-2.0,-1.0)$ \\
\hline Vegetable portions & $2.7(1.4)$ & $2.8(1.5)$ & $0.1(-1.0,1.0)$ & 26.0 & $1.5(1.0,3.0)$ & 21.0 & $-1.3(-2.0,-1.0)$ \\
\hline Eats breakfast (\%) & 77.4 & 78.3 & & - & - & - & - \\
\hline Worried about control (\%) & 28.0 & 36.2 & & - & - & - & - \\
\hline \multicolumn{8}{|l|}{ US replication cohort } \\
\hline \multicolumn{8}{|l|}{ Anthropometrics } \\
\hline Weight (kg) & $78.7(20.0)$ & $78.3(19.5)$ & $-0.4(-6.8,5.0)$ & 44.0 & $3.9(0.9,7.7)$ & 40.0 & $-5.3(-10.9,-1.2)$ \\
\hline $\mathrm{BMI}\left(\mathrm{kg} \mathrm{m}^{-2}\right)$ & $28.2(6.5)$ & $28.0(6.4)$ & $-0.1(-2.4,1.9)$ & 44.0 & $1.4(0.3,2.8)$ & 40.0 & $-1.9(-3.8,-0.4)$ \\
\hline \multicolumn{8}{|l|}{ LSF-FFQ diet questionnaire } \\
\hline DQS & $10.6(1.8)$ & $10.8(1.7)$ & $0.2(-0.2,3.0)$ & 37.0 & $1.9(1.0,3.0)$ & 31.0 & $-1.7(-3.0,-1.0)$ \\
\hline Fruit portions & $1.7(1.6)$ & $1.8(1.8)$ & $0.1(-1.0,1.0)$ & 24.0 & $1.7(1.0,2.0)$ & 20.0 & $-1.5(-2.0,-1.0)$ \\
\hline Vegetable portions & $2.3(1.4)$ & $2.3(1.5)$ & $0.1(-1.0,1.0)$ & 24.0 & $1.5(1.0,2.5)$ & 22.0 & $-1.3(-2.0,-1.0)$ \\
\hline Glasses of juice & $0.4(1.3)$ & $0.4(1.4)$ & $0.0(0.0,0.0)$ & 8.0 & $2.3(0.5,5.0)$ & 9.0 & $-1.9(-3.0,-0.5)$ \\
\hline \multicolumn{8}{|l|}{ Activity behaviours } \\
\hline Weekday sleep (h) & $7.0(1.1)$ & $7.1(1.0)$ & $0.1(-1.0,1.0)$ & 20.0 & $1.2(1.0,2.0)$ & 15.0 & $-1.3(-2.0,-1.0)$ \\
\hline Weekend sleep (h) & $7.6(1.2)$ & $7.5(1.1)$ & $0.0(-1.0,1.0)$ & 13.0 & $1.3(1.0,2.0)$ & 16.0 & $-1.3(-2.0,-1.0)$ \\
\hline \multicolumn{8}{|l|}{ Eating behaviour } \\
\hline Number of main meals & $1.9(1.2)$ & $1.9(1.2)$ & $-0.1(-1.0,0.0)$ & 5.0 & $1.0(1.0,1.0)$ & 15.0 & $-1.1(-1.0,-1.0)$ \\
\hline Number of snacks & $2.1(0.6)$ & $2.0(0.6)$ & $-0.1(-1.0,1.0)$ & 13.0 & $1.4(1.0,2.0)$ & 27.0 & $-1.4(-2.0,-1.0)$ \\
\hline Eats breakfast (\%) & 77.7 & 77.0 & & - & - & - & - \\
\hline Worried about control (\%) ${ }^{\mathrm{b}}$ & 22.1 & 33.2 & & - & - & - & - \\
\hline
\end{tabular}

The data are presented as means (s.d.) of percentage increase/decrease and size of change unless otherwise stated. a Not including breakfast. ${ }^{b}$ Worried about losing control of eating. 


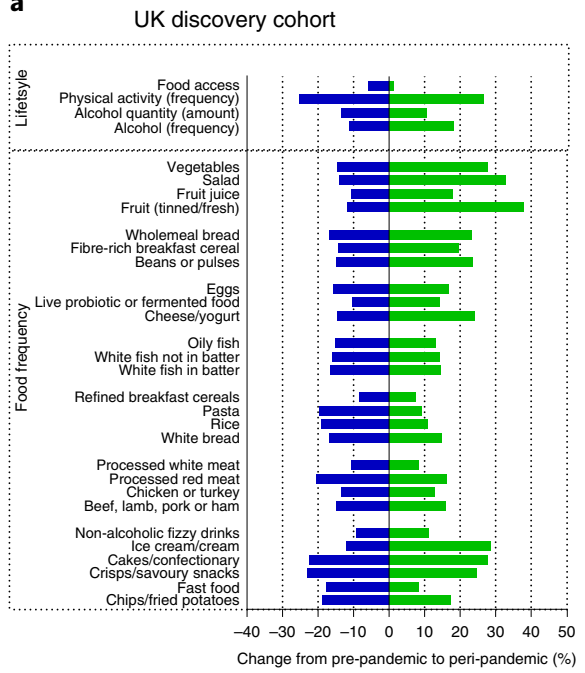

b

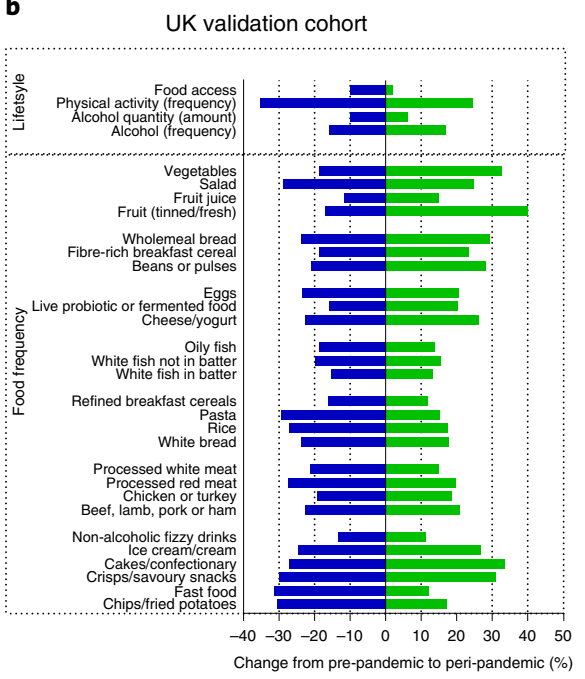

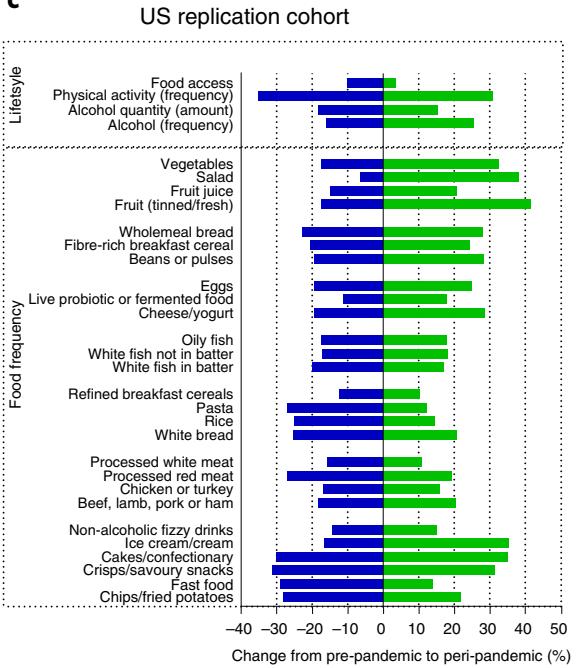

Fig. 3 | Proportion of the population changing the frequency of intake of food groups and behaviours. a-c, Percentage of the cohort that changed (increased/decreased) the frequency of dietary intake and lifestyle behaviours from pre-pandemic to peri-pandemic, obtained from the amended LSF-FFQ and others, in the UK discovery $(n=201,301)$ (a), UK validation $(n=75,840)(\mathbf{b})$ and US replication $(n=14,473)$ cohorts (c). The data are provided in Supplementary Table 6.

pandemic, we undertook a factor analysis (Methods) identifying two distinct diet and lifestyle patterns labelled as 'healthier' and 'less healthy' on the basis of items and loadings summarized in Fig. 4 and Supplementary Table 8.

Participants' demographic and behavioural characteristics differed between quartiles ( $n=50,497$ per quartile) of the diet and lifestyle patterns (Supplementary Tables 9 and 10). In the United Kingdom, those with the highest adherence to the 'healthier' pattern (quartile 4 (Q4) versus Q1) were on average older (61 versus 53 years), less likely to smoke (1.2\% versus $3.9 \%)$ and more likely to reside in an area with a lower level of deprivation $(44.8 \%$ versus $37.7 \%$ with IMD 8-10) than those with the lowest adherence (all $P<0.001$ ) (Table 3). A broadly similar pattern was observed in the US replication cohort (Supplementary Table 9).

Individuals most adherent to the 'less healthy' pattern lost more weight than those reporting higher adherence to the 'healthier' pattern (by $1.1 \mathrm{~kg}$ ) (Table 3). The same trends of association were observed in other diet and lifestyle measures (for both the UK and US cohorts), with larger increases in weeknight sleep, DQS, and fruit and vegetable consumption and a larger decrease in the number of snacks among those most adherent to the 'less healthy' pattern compared with the 'healthier' lifestyle pattern. These findings suggest that participants with less healthy behaviours in the pre-pandemic phase were more likely to implement healthy changes. This may be a consequence of a greater scope for improvement in the 'less healthy' group, while those classified as having a healthy pre-pandemic lifestyle pattern tended to retain their beneficial health behaviours and experienced minimal change. This observation was also reflected in those with a lower DI typically being highly adherent to the 'healthier' pattern while those with a higher DI tended to be adherent to the 'less healthy' pattern (both $P<0.001$ ).

To assess whether the population demographics were driving this finding, in the United Kingdom, we stratified individuals most adherent to the 'less healthy' pattern according to their community-level deprivation, age, sex and constituent countries. When stratified according to deprivation (IMD 1-3 versus 8-10), the improvement in diet quality ( 0.92 versus 0.94 units) and reduction in body weight $(-0.71$ versus $-0.99 \mathrm{~kg})$ remained, although attenuated. Age, sex and country also had only small impacts on the change in weight (aged 18-25 yr, $-1.46 \mathrm{~kg}$; versus aged $>65 \mathrm{yr}$, $-0.58 \mathrm{~kg}$; male, $-1.16 \mathrm{~kg}$; versus female, $-0.70 \mathrm{~kg}$; England, $-0.89 \mathrm{~kg}$; versus Wales, $-0.81 \mathrm{~kg}$; versus Scotland, $-0.69 \mathrm{~kg}$; versus Northern Ireland, $-0.43 \mathrm{~kg}$ ) and minimal impacts on DQS (aged 18-25 yr, 0.88 ; versus aged $>65 \mathrm{yr}, 0.82$; England, 0.94; versus Wales, 0.98; versus Scotland, 0.90; versus Northern Ireland, 0.70). Therefore, irrespective of community-level deprivation, age, sex and location, individuals most adherent to a 'less healthy' pre-pandemic pattern experienced beneficial changes in diet quality and weight. Consequentially, our data suggest apparent positive diet and lifestyle changes related to the COVID-19 pandemic for a large proportion of the study demographic.

\section{Discussion}

The changes in daily routine due to national and local measures in response to the COVID-19 pandemic could be viewed as a unique natural experiment of lifestyle disruption, health behaviour change and associated longer-term health outcomes for individuals and populations. We have described the impact of the pandemic on lifestyle behaviour changes in samples of the UK and US populations using data from the largest survey of diet and lifestyle during the pandemic to date.

The disruption of health behaviours by the pandemic, which was more pronounced in females, younger participants and those residing in more deprived areas, was associated with a higher and more variable bidirectional change in body weight. This observation is a likely consequence of unpaid care and family responsibilities falling disproportionately to women, particularly younger females, with women over-represented in lower-income households ${ }^{34}$.

Although we observed no mean changes in diet and lifestyle behaviours in the total population, considerable inter-individual variation exists upon stratifying the data. This variability in the responses of individual health behaviours and weight changes to COVID-19, in concert with minimal mean population change, is in agreement with studies exploring the impact of comparable mandatory public health interventions across Europe ${ }^{5,6,35,36}$. While direct comparisons between countries can prove difficult due to differences in populations, government lockdown strategies and dietary habits (among other factors), these results suggest some similarities in terms of behaviour change. 


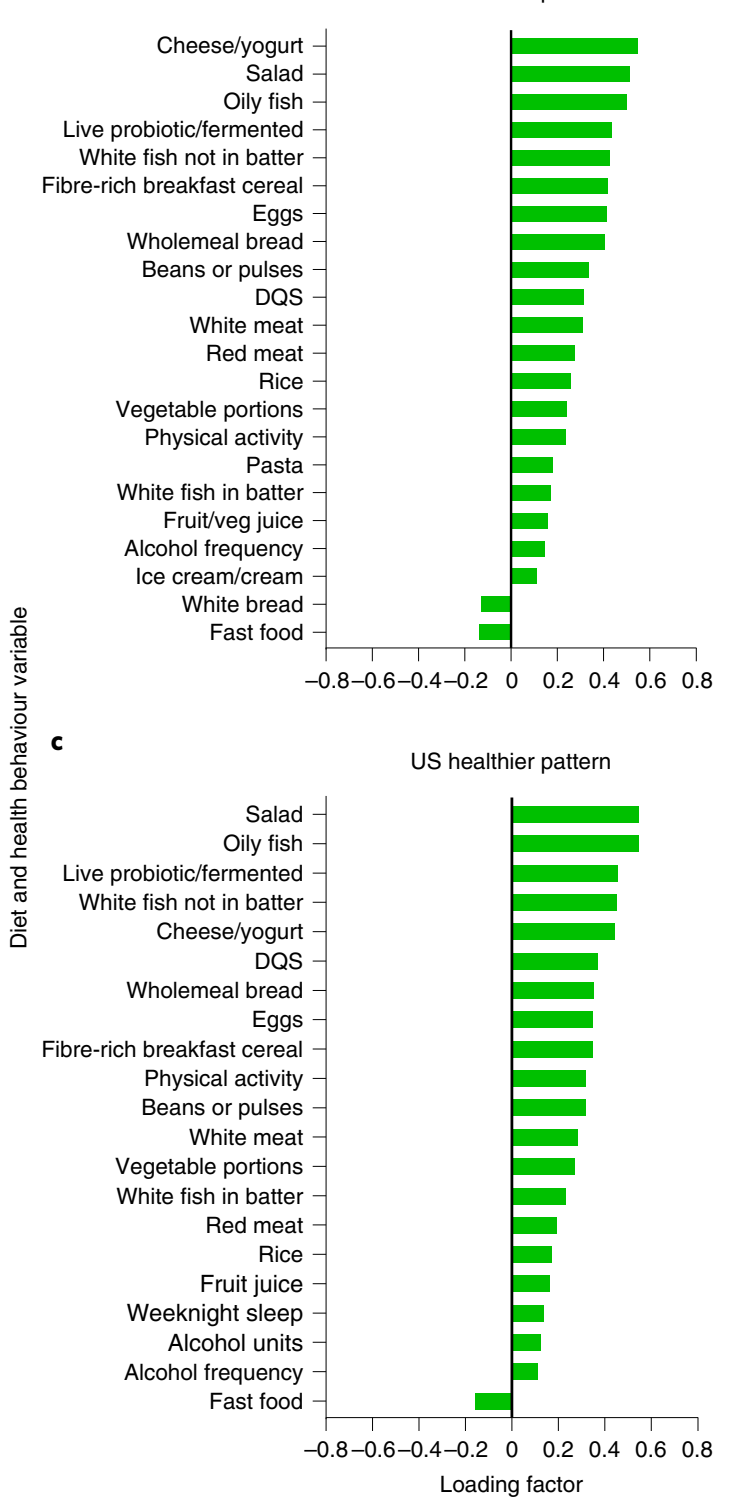

b

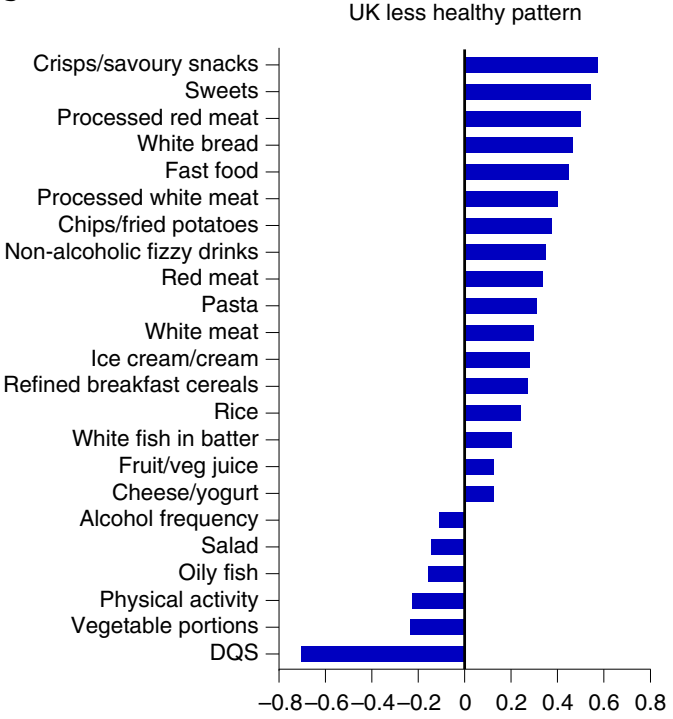

d

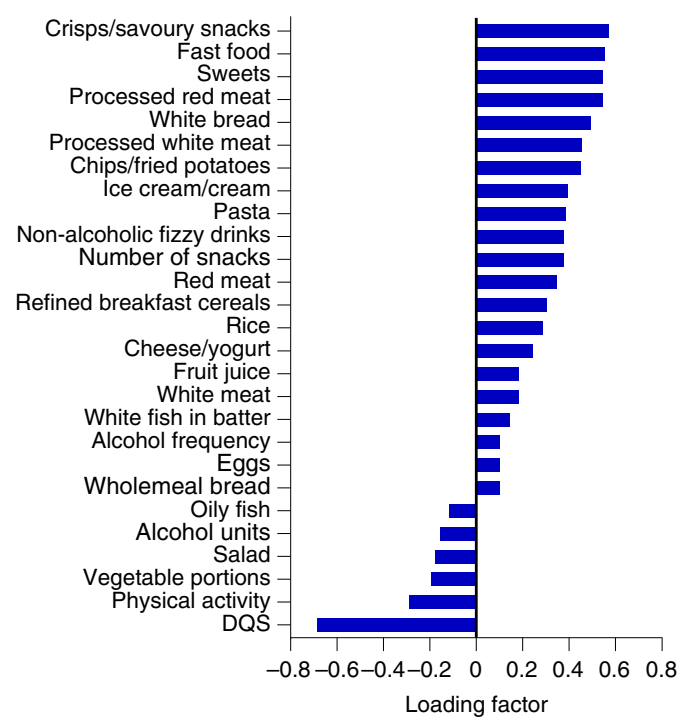

Fig. 4 | Loadings of diet and health behaviour variables to 'healthier' and 'less healthy' clusters. a-d, Loadings ( $\geq 0.1$ ) for 'healthier' (a) and 'less healthy' (b) patterns in the UK discovery cohort $(n=201,301)$ and 'healthier' (c) and 'less healthy' (d) patterns in the US replication cohort ( $n=14,473)$. A full breakdown of the loading of each diet and health behaviour variable for the 'healthier' and 'less healthy' patterns can be seen in Supplementary Table 8.

Most surprisingly, those participants who were identified to have a 'less healthy' pattern pre-pandemic were more likely to experience improvements in diet quality and greater weight loss peri-pandemic, irrespective of deprivation status. While this may reflect some bias due to the users of the app being self-selected and typically of a higher socio-economic demographic than the average UK and US citizen, the NutriNet-Sante study also observed that positive health behaviour changes were associated with less healthy pre-pandemic behaviours 5 . Therefore, for a significant proportion of the population, the pandemic may not have had the detrimental impact on diet and lifestyle behaviours that has previously been speculated and reported in the media ${ }^{37,38}$.

Excess body weight has been linked with an increase of COVID-19 severity ${ }^{39,40}$ and chronic disease mortality ${ }^{41}$ and is known to be interrelated with social determinants of health, including systematic racism ${ }^{42}$ and disparities in food security and socioeconomic status ${ }^{43,44}$. Bidirectional weight changes were observed between groups stratified by levels of deprivation, with those reporting less healthy behaviours and residing in more deprived areas experiencing more weight gain than those residing in less deprived areas. However, irrespective of deprivation, physical activity and diet quality were identified as key determinants of short-term weight gain and loss. It is noteworthy that the mean change in body weight was greater than typical daily body weight fluctuations ${ }^{45}$ and, for some, a weight loss of a magnitude to confer improved metabolic function ${ }^{46}$.

As highlighted in the Foresight Report, weight gain and obesity are the result of complex system interaction ${ }^{47}$; it therefore may be reasonable to assume that the COVID-19 pandemic has disrupted many active components across multiple domains. From a public health perspective, future research questions should focus on identifying the higher-level disruptions that impact individual health behaviours-for example, working from home, reduced commuting, food availability, structural inequalities or societal influences. 
Table 3 | Participant characteristics and lifestyle changes according to pre-pandemic health behaviour

\begin{tabular}{|c|c|c|c|c|}
\hline & \multicolumn{4}{|c|}{ UK discovery cohort } \\
\hline & \multicolumn{2}{|c|}{ Less healthy pattern } & \multicolumn{2}{|c|}{ Healthier pattern } \\
\hline & Q1 & Q4 & Q1 & Q4 \\
\hline Male/female (\%) & $21.3 / 78.7$ & $37.4 / 62.6$ & $29.8 / 70.2$ & $31.0 / 69.0$ \\
\hline Age (yr) & $61(53,68)$ & $53(42,62)$ & $53(42,62)$ & $61(53,68)$ \\
\hline \multicolumn{5}{|l|}{ Preconditions } \\
\hline Diabetes & 3.1 & 4.8 & 4.9 & 3.2 \\
\hline Heart disease & 3.6 & 3.4 & 3.3 & 4.2 \\
\hline Kidney disease & 0.8 & 1.1 & 1.1 & 0.8 \\
\hline Wales & 3.8 & 5.3 & 5.2 & 4.3 \\
\hline Northern Ireland & 0.4 & 0.7 & 0.6 & 0.6 \\
\hline \multicolumn{5}{|l|}{ IMD } \\
\hline $1-3$ & 7.7 & 10.7 & 12.1 & 6.9 \\
\hline $4-7$ & 35.2 & 34.0 & 34.8 & 34.2 \\
\hline $8-10$ & 43.9 & 39.5 & 37.8 & 44.9 \\
\hline \multicolumn{5}{|l|}{ DI } \\
\hline 0 & 13.5 & 6.0 & 7.9 & 11.4 \\
\hline 1 & 28.7 & 19.0 & 20.7 & 28.2 \\
\hline 4 & 8.7 & 16.7 & 15.1 & 9.5 \\
\hline Fruit portions & $0.04(-1.0,1.0)$ & $0.20(-1.0,1.0)$ & $0.18(-1.0,1.0)$ & $0.05(-1.0,1.0)$ \\
\hline Vegetable portions & $-0.02(-1.0,1.0)$ & $0.19(-1.0,1.0)$ & $0.15(-1.0,1.0)$ & $-0.01(-1.0,1.0)$ \\
\hline \multicolumn{5}{|l|}{ Eating behaviour } \\
\hline \multirow[t]{4}{*}{ Number of snacks } & $0.06(-1.0,1.0)$ & $-0.33(-2.0,1.0)$ & $-0.15(-1.0,1.0)$ & $-0.06(-1.0,1.0)$ \\
\hline & \multicolumn{4}{|c|}{ US replication cohort } \\
\hline & \multicolumn{2}{|c|}{ Less healthy pattern } & \multicolumn{2}{|c|}{ Healthier pattern } \\
\hline & Q1 & Q4 & Q1 & Q4 \\
\hline \multicolumn{5}{|l|}{ Basic characteristics } \\
\hline Male/female (\%) & $12.8 / 87.1$ & $22.4 / 77.5$ & $17.1 / 82.8$ & $18.3 / 81.7$ \\
\hline Age (yr) & $63(53,69)$ & $56(41,67)$ & $55(39,66)$ & $63(54,70)$ \\
\hline Race: white & 90.1 & 87.2 & 87.1 & 88.4 \\
\hline Smokers & 1.6 & 4.8 & 4.4 & 0.0 \\
\hline \multicolumn{5}{|l|}{ Preconditions } \\
\hline Diabetes & 3.5 & 7.4 & 7.0 & 4.2 \\
\hline Heart disease & 5.7 & 6.6 & 5.9 & 6.1 \\
\hline Kidney disease & 1.5 & 2.1 & 2.3 & 1.5 \\
\hline & & & & Continued \\
\hline
\end{tabular}


Table 3 | Participant characteristics and lifestyle changes according to pre-pandemic health behaviour (Table continued)

US replication cohort

\begin{tabular}{llllll}
\hline \multicolumn{2}{c}{ Less healthy pattern } & & \multicolumn{3}{c}{ Healthier pattern } \\
\cline { 1 - 1 } & Q4 & Q1 & Q4
\end{tabular}

\section{Changes}

Anthropometrics

Weight (kg)

$-0.06(-5.9,5.0$

Diet

\section{DQS}

Fruit portions

Vegetable portions

\section{Eating behaviour}

Number of snacks
$0.97(-4.5,5.9)$

$-2.0(-10.1,4.6)$

$-0.76(-8.6,5.9)$

$\begin{array}{ll}-0.48(-2.0,1.0) & 1.14(-1.0,4.0) \\ -0.02(-1.0,1.0) & 0.21(-1.0,1.0) \\ -0.15(-1.0,1.0) & 0.29(-1.0,2.0)\end{array}$

$-0.42(-2.0,1.0)$

$0.22(-1.0,1.0)$

$$
\begin{aligned}
& 0.53(-1.0,3.0) \\
& 0.19(-1.0,1.0) \\
& 0.21(-1.0,1.0)
\end{aligned}
$$$$
\text { Q4 }
$$

$-0.16(-2.0,2.0)$
$-0.01(-1.0,1.0)$
$-0.07(-1.0,1.0)$

$-0.02(-1.0,1.0)$

Upper (Q4) and lower (Q1) quartiles most adherent to 'less healthy' and 'healthier' patterns, clustered using principal component analysis. Changes are shown as mean (10th percentile, 90 th percentile). For each quartile, $n=50,497$ (UK) and 3,684 (US).

These pose the following questions: if we can identify facilitators and barriers to health behaviour changes, how sustainable are these changes? Furthermore, if body weight changes are maintained in the long term, are they associated with corresponding improvements in metabolic health?

While this study had multiple strengths, including sample size, an independent replication cohort and longitudinal data, we note several limitations. The first is the self-reported nature of the data collected, with the potential of recall bias and the unknown impact of COVID-19 on retrospective data collection ${ }^{48}$. Further limitations include the data collection methods, which were modified part-way through the study to minimize participant burden and decrease attrition (Methods), although sensitivity analysis displayed minimal population characteristic differences. Additionally, the DI is a crude bidirectional index composed of only five behaviours. Further work is needed to determine how the direction of change in the individual components may contribute to health outcomes. Considerations for future studies would be to collect data on level of isolation, mental health, comorbidities, job role and furlough status to assist in the interpretation of lifestyle behaviour changes in response to COVID-19. The generalizability of our findings to a wider population sample also needs to be determined, given the differences in age, IMD and ethnicity of the ZOE COVID Study cohort compared with the general population. Regression to the mean can be problematic in studies that focus on assessing changes in behaviours in subgroups of the population where only a single follow-up assessment is made. Thus, to some extent, the improvements in health behaviours seen in people recording the least healthy behaviours at baseline could be artificial improvements. Nevertheless, the same degree of change was not observed in people who reported the most healthy baseline behaviours, reinforcing the argument that changes in health seen in either group are likely to be real.

In conclusion, this large population-based study has demonstrated that the COVID-19 pandemic has impacted individuals differently, with those most disrupted being more susceptible to weight change.

\section{Methods}

Study design and participants. The ZOE COVID Symptom Study mobile application was developed by the technology company ZOE in collaboration with King's College London, UK, and Massachusetts General Hospital, USA. It was made available for free download for Apple and Android users (on 24 March 2020 in the United Kingdom and on 29 March 2020 in the United States). The application offers a guided interface developed using Expo and ReactNative (JavaScript). The participants were requested to report information including baseline demographics, comorbidities, daily symptoms and COVID-19 testing outcomes.

Using the ZOE COVID Study app, a longitudinal, retrospective, observational cohort study investigating diet quality, diet habits and lifestyle changes before and during the pandemic was launched (from 31 July to 25 September 2020 in the United Kingdom and from 25 September to 30 November 2020 in the United States). The participants were recruited via the app interface. At enrolment, the participants consented to the use of their information for research investigations related to COVID-19, diet and lifestyle, and to applicable privacy policies and terms of use. The study was approved by Partners Human Research Committee (protocol no. 2020P000909) and King's College London ethics committee (REMAS ID 18210; LRS-19/20-18210). The ZOE COVID Study app is registered with ClinicalTrials.gov, NCT04331509. Data collected in the app are being shared with other health researchers through the NHS-funded Health Data Research UK (HDRUK)/SAIL Consortium, housed in the UK Secure e-Research Platform in Swansea, Wales. Anonymized data are available to be shared with bona fide researchers through HDRUK according to their protocols in the public interest. US investigators are encouraged to coordinate data requests through the Coronavirus Pandemic Epidemiology (COPE) Consortium (www.monganinstitute.org/ cope-consortium)

Procedures. Information obtained through the ZOE COVID Study mobile application has been described in detail previously ${ }^{23,49}$. The participants were requested to complete a retrospective questionnaire investigating diet quality, diet habits and lifestyle at two time points: (1) peri-pandemic, described as the previous month (from user access), and (2) pre-pandemic, described as the month of February 2020. The sections contained identical sets of questions collated by the research team and included the validated LSF-FFQ ${ }^{25}$ developed by Cleghorn and listed in the Nutritools (www.nutritools.org) library (Supplementary Information).

Questionnaire userface. The user experience was drafted in a requirements document by the engineering and product team at ZOE. A designer laid out a format optimized for mobile and tablet devices. The format and the requirements document were reviewed by the researchers. The answer options were developed using a library of standard form components, including a dropdown list, multi-select checkboxes and user input validation. Interstitial pages provided guidance to the users on how to answer these questions. A prominent callout label displayed the time period at the top of every screen to remind users to 'Answer for the last 4 weeks' or 'Answer for the month of February 2020'.

Prior to launching, the release candidate of the app (including the questionnaire) was circulated to internal quality assurance testers and nutrition researchers to check that the delivered software matched the requirements document. The sample data collected from the quality assurance sessions were reviewed and checked to be in an analysable format. The questionnaire was launched via controlled roll-out to a smaller cohort of users before wider dissemination. Existing users were invited to complete the questionnaire using a feature flag, which allows the targeting of specific users before roll-out to a wider user base. A total of 199,230 UK users and 19,079 US users declined the diet study invite in the app.

The participants were prompted to complete an additional consent form to allow processing of data for purposes outside of COVID-19 research, to allow for further investigations into diet and lifestyle aspects independent of COVID-19. As users submit questionnaires to the application, the data are submitted in JSON 
format to REST APIs / WebServers that store the questionnaire responses in a Postgres Database that is hosted in the Google Cloud Platform operated by ZOE. These data are anonymized and exported daily into comma-separated value files that are delivered via Secure FTP from ZOE to the research partners for further analysis.

The LSF-FFQ interface was developed by the engineering team in conjunction with the designer using a four-by-two grid of the eight FFQ 'frequency of consumption' options. Effort was made to retain the original structure and layout of the paper-based validated LSF-FFQ ${ }^{25}$. The questionnaire was designed for participants to complete the first section in reference to the previous four weeks (peri-pandemic) and then to complete an identical section for the month of February 2020 (pre-pandemic). Additional food items were included in the LSF-FFQ under general consensus by two dietitians and a nutritionist. These food items included fast food; eggs and egg dishes; refined carbohydrates such as white rice, white pasta and white bread; and live probiotic or fermented foods such as kimchi, live yogurt and kefir (Supplementary Information).

Data collection. Data were collected via two similar app flows in the United Kingdom (over an eight-week period) and via one app flow in the United States (over a nine-week period). In the United Kingdom, the questionnaire was released to $1 \%$ of users on 31 July 2020, 2\% on 4 August 2020 and $100 \%$ on 6 August 2020 . In this first app flow, 380,847 participants completed the questionnaire. After reviewing feedback from UK users, the app flow was altered to limit participant burden and to reduce attrition. On 7 August 2020, a new question was added after the participants had completed the first peri-pandemic section asking 'Has your diet changed?' ('Yes', 'No' or 'Unsure'). If users answered 'yes' or 'unsure', they were invited to complete the pre-pandemic section. If users answered 'No', the app flow ended (Extended Data Fig. 1). A total of 448,321 participants participated in the second flow. In the United States, the questionnaire was launched to $20 \%$ of users on 3 September 2020 and $100 \%$ on 28 September $2020(n=67,118$ via the second flow only). The mode completion time for both sections was 14-16 minutes. Within the app, individuals reported their height and weight in response to the questions 'About how much do you weigh without shoes?' and 'About how tall are you without shoes?'. These were converted (if necessary) to kilograms and metres before calculating the BMI $\left(\mathrm{kg} \mathrm{m}^{-2}\right)$. Participant characteristics are described in Supplementary Table 1 .

Exclusions and cohort interaction analysis. Participants were excluded if (1) the primary outcome (BMI) was below the 0.1 st percentile or above the 99.9 th percentile (excluding $10-150 \mathrm{~kg} \mathrm{~m}^{-2}$ ), (2) they were pregnant, (3) they were less than 18 years old, or (4) they left questionnaire sections incomplete (the full details are given in Supplementary Table 11). Additional exclusions were made per analysis, with the application of percentiles on the consensus of the research team (S.B., R.G., M.M. and E.R.L.). The participants were divided into cohorts relative to the flow of the app (Extended Data Fig. 1) and by country: the discovery cohort $(n=380,847)$ from the first flow, the UK validation cohort $(n=448,321)$ from the second flow ('Has your diet changed') and the US replication cohort $(n=67,118)$. A sensitivity analysis was performed to assess significant population differences between the discovery and validation cohorts. We defined 'change' and 'no change' groups within both cohorts relative to a $>1$-point multi-directional change in DQS (no change, $\leq 1$ and $\geq-1$ ). Demographic characteristics were compared between the 'change' and 'no change' groups (Supplementary Table 2). The interaction was plotted between the primary outcome (BMI) and the primary exposure (DQS). To minimize bias, all analyses in this study were performed using the discovery cohort. The DI as well as diet and lifestyle pattern analyses were replicated in the UK validation and US replication cohorts.

DI. A diet and lifestyle DI was developed. The domains sleep (on weekdays), physical activity, diet (DQS and snacking frequency) and alcohol intake frequency were selected on the basis of commonality in previously published healthy lifestyle scores $^{50,51}$. A bidirectional change was determined as one point for any change (either positive or negative) for each variable, with the maximum disruption score as 5 and the minimum as 0 (Supplementary Table 3 )

Pre- and peri-pandemic comparisons. In the discovery cohort, and validated in the UK validation cohort, the mean, s.d., and 10th and 90th percentiles for the pre-pandemic and peri-pandemic periods, the percentage change (between the two time points), and the number of participants who increased/decreased were applied to the continuous and categorical variables (Supplementary Tables 4, 6 and 7). For the continuous variables, the quantity of increase/decrease was also described. Changes in body weight, BMI, DI, physical activity, alcohol intake (units), snacking, fruit and vegetable intake (combined) and diet quality were visualized across UK geographical regions using the Python package geopandas v.0.7.0. IMD was estimated according to small geographical location, or neighbourhood, ranking areas according to multiple deprivation parameters (least deprived $=32,844$, most deprived $=1$ ). Further reference to deprivation throughout is related to IMD status ${ }^{24}$. Deciles for England, Wales and Northern Ireland were pre-generated by the official data source ${ }^{24}$. For Scotland, the same deciles were applied by the research team.
Factor analysis. Factor analysis with orthogonal transformation (varimax procedure) was used to construct a distinct 'healthier' diet and lifestyle pattern and a 'less healthy' dietary pattern (the loadings and input variables are depicted in Supplementary Table 8). The dietary patterns were stratified into quartiles to compare demographic characteristics and body weight changes (Supplementary Tables 9 and 10).

Structural equation modelling. Structural equation modelling was used to describe the relationship of exposures of changes in sleep (on weekends and weekdays), physical activity, diet (DQS and snacking frequency) and alcohol intake (frequency and quantity) (Supplementary Table 5). Age was included in the model owing to its established relationship with $\mathrm{BMI}^{32}$. Changes in body weight were categorized as (1) an absolute bidirectional change, (2) an increase in body weight or (3) a decrease in body weight from pre-pandemic to peri-pandemic weight status. Furthermore, stratified models were also developed on the basis of a low and high IMD. The model was fitted under a maximum likelihood framework using covariance matrices. Relative model fit was assessed using the comparative fit index $(0 \text {, no fit; } 1 \text {, perfect fit })^{52}$, and the absolute fit by the root mean square error of approximation. Statistical analysis was performed using SPSS (IBM Corp), R v.3.5.1 (Foundation for Statistical Computing; https://www.R-project.org/) and $\mathrm{AMOS}^{53}$.

Ethics. The King's College London Ethics Committee approved the ethics for the app, and all users provided consent for non-commercial use. An informal consultation with TwinsUK members over email and social media before the app was launched found that they were overwhelmingly supportive of the project. The US protocol was approved by the Partners Human Research Committee.

\section{Data availability}

Deidentified participant baseline data collected in the ZOE COVID Study smartphone app can be shared with other health researchers through the NHS-funded Health Data Research UK and Secure Anonymised Information Linkage consortium, housed in the UK Secure Research Platform (Swansea, UK). Anonymized data are available to be shared with researchers according to their protocols in the public interest (https://web.www.healthdatagateway.org/ dataset/fddcb382-3051-4394-8436-b92295f14259). Researchers must apply to gain access through Health Data Research UK. The data from the Diet and Lifestyle Questionnaire can be made available by application to TREC at the Department of Twins Research and Epidemiology at King's College London. US investigators are encouraged to coordinate data requests through the COPE Consortium (www.monganinstitute.org/cope-consortium). Data updates can be found at https://covid.joinzoe.com.

\section{Code availability}

The app code is publicly available at https://github.com/zoe/ covid-tracker-react-native.

Received: 28 January 2021; Accepted: 28 September 2021; Published online: 16 December 2021

\section{References}

1. Groarke, J. M. et al. Loneliness in the UK during the COVID-19 pandemic: cross-sectional results from the COVID-19 Psychological Wellbeing Study. PLOS ONE 15, e0239698 (2020).

2. Hwang, T.-J., Rabheru, K., Peisah, C., Reichman, W. \& Ikeda, M. Loneliness and social isolation during the COVID-19 pandemic. Int. Psychogeriatr. 32, 1217-1220 (2020).

3. Bell, T., Gardiner, L. \& Tomlinson, D. Getting Britain Working (Safely) Again The Next Phase of the Coronavirus Job Retention Scheme (Resolution Foundation, 2020).

4. Mardones, F. O. et al. The COVID-19 pandemic and global food security. Front. Vet. Sci. 7, 578508 (2020).

5. Deschasaux-Tanguy, M. et al. Diet and physical activity during the COVID-19 lockdown period (March-May 2020): results from the French NutriNet-Sante cohort study. Am. J. Clin. Nutr. 113, 924-938 (2020).

6. Renzo, L. D. et al. Eating habits and lifestyle changes during COVID-19 lockdown: an Italian survey. J. Transl. Med. 18, 229 (2020).

7. Sidor, A. \& Rzymski, P. Dietary choices and habits during COVID-19 lockdown: experience from Poland. Nutrients 12, 1657 (2020).

8. Just-Østergaard, E., Mortensen, E. L. \& Flensborg-Madsen, T. Major life events and risk of alcohol use disorders: a prospective cohort study. Addiction 113, 25-33 (2018).

9. Rowshan Ravan, A., Bengtsson, C., Lissner, L., Lapidus, L. \& Björkelund, C. Thirty-six-year secular trends in sleep duration and sleep satisfaction, and associations with mental stress and socioeconomic factors-results of the Population Study of Women in Gothenburg, Sweden. J. Sleep Res. 19, 496-503 (2010) 
10. Li, Y. et al. Relationship between stressful life events and sleep quality: rumination as mediator and resilience as a moderator. Front. Psychiatry 10, 348 (2019).

11. Sims, R. et al. Perceived stress and eating behaviors in a community-based sample of African Americans. Eat. Behav. 9, 137-142 (2008).

12. Schweren, L. J. S. et al. Diet quality, stress and common mental health problems: a cohort study of 121,008 adults. Clin. Nutr. https://doi.org/ 10.1016/j.clnu.2020.06.016 (2020).

13. Stults-Kolehmainen, M. A. \& Sinha, R. The effects of stress on physical activity and exercise. Sports Med. 44, 81-121 (2014).

14. Geiker, N. R. W. et al. Does stress influence sleep patterns, food intake, weight gain, abdominal obesity and weight loss interventions and vice versa? Obes. Rev. 19, 81-97 (2018).

15. Mehlig, K. et al. Negative life events predict weight gain in a 13-year follow-up of an adult Swedish population. J. Psychosom. Res. 132, 109973 (2020).

16. Daivadanam, M. et al. Lifestyle change in Kerala, India: needs assessment and planning for a community-based diabetes prevention trial. BMC Public Health 13, 95 (2013).

17. Wing, R. R. \& Phelan, S. Long-term weight loss maintenance. Am. J. Clin. Nutr. 82, 222S-225S (2005).

18. Fothergill, E. et al. Persistent metabolic adaptation 6 years after "The Biggest Loser" competition. Obesity 24, 1612-1619 (2016).

19. González-Muniesa, P. et al. Obesity. Nat. Rev. Dis. Primers 3, 17034 (2017).

20. Gill, T. Importance of preventing weight gain in adulthood. Asia Pac. J. Clin. Nutr. 11, S632-S636 (2002).

21. Obesity: Preventing and Managing the Global Epidemic (WHO, 2015).

22. Naja, F. \& Hamadeh, R. Nutrition amid the COVID-19 pandemic: a multi-level framework for action. Eur. J. Clin. Nutr. 74, 1117-1121 (2020).

23. Drew, D. A. et al. Rapid implementation of mobile technology for real-time epidemiology of COVID-19. Science 368, 1362-1367 (2020).

24. English Indices of Deprivation 2015 (Ministry of Housing \& Communities \& Local Government, 2015).

25. Cleghorn, C. L. et al. Can a dietary quality score derived from a short-form FFQ assess dietary quality in UK adult population surveys? Public Health Nutr. 19, 2915-2923 (2016).

26. Yin, J. et al. Relationship of sleep duration with all-cause mortality and cardiovascular events: a systematic review and dose-response meta-analysis of prospective cohort studies. J. Am. Heart Assoc. 6, e005947 (2017).

27. Aune, D. et al. Fruit and vegetable intake and the risk of cardiovascular disease, total cancer and all-cause mortality-a systematic review and dose-response meta-analysis of prospective studies. Int. J. Epidemiol. 46 1029-1056 (2017).

28. Wood, A. M. et al. Risk thresholds for alcohol consumption: combined analysis of individual-participant data for 599912 current drinkers in 83 prospective studies. Lancet 391, 1513-1523 (2018).

29. Kyu, H. H. et al. Physical activity and risk of breast cancer, colon cancer, diabetes, ischemic heart disease, and ischemic stroke events: systematic review and dose-response meta-analysis for the Global Burden of Disease Study 2013. BMJ 354, i3857 (2016).

30. Wang, Y.-Q. et al. Increased eating frequency is associated with lower obesity risk, but higher energy intake in adults: a meta-analysis. Int. J. Environ. Res. Public Health 13, 603 (2016).

31. O'Connor, L., Brage, S., Griffin, S. J., Wareham, N. J. \& Forouhi, N. G. The cross-sectional association between snacking behaviour and measures of adiposity: the Fenland Study, UK. Br. J. Nutr. 114, 1286-1293 (2015).

32. Lloyd-Sherlock, P. et al. Population ageing and health. Lancet 379 , 1295-1296 (2012).

33. Wang, X. et al. Fruit and vegetable consumption and mortality from all causes, cardiovascular disease, and cancer: systematic review and dose-response meta-analysis of prospective cohort studies. BMJ 349, g4490 (2014).

34. Women shoulder the responsibility of 'unpaid work' (ONS, 2016); https://www.ons.gov.uk/employmentandlabourmarket/peopleinwork/ earningsandworkinghours/articles/womenshouldertheresponsibility ofunpaidwork/2016-11-10

35. Di Renzo, L. et al. Psychological aspects and eating habits during COVID-19 home confinement: results of EHLC-COVID-19 Italian Online Survey. Nutrients 12, 2152 (2020).

36. Scarmozzino, F. \& Visioli, F. COVID-19 and the subsequent lockdown modified dietary habits of almost half the population in an Italian sample. Foods 9, 675 (2020).

37. How coronavirus lockdown will make us fatter. Daily Mail (3 April 2020).

38. Clemmensen, C., Petersen, M. B. \& Sørensen, T. I. A. Will the COVID-19 pandemic worsen the obesity epidemic? Nat. Rev. Endocrinol. 16, 469-470 (2020).

39. Kalligeros, M. et al. Association of obesity with disease severity among patients with coronavirus disease 2019. Obesity 28, 1200-1204 (2020).
40. Goossens, G. H. et al. Obesity and COVID-19: a perspective from the European Association for the Study of Obesity on immunological perturbations, therapeutic challenges, and opportunities in obesity. Obes. Facts 13, 439-452 (2020).

41. Hruby, A. \& Hu, F. B. The epidemiology of obesity: a big picture. Pharmacoeconomics 33, 673-689 (2015).

42. Laurencin, C. T. \& McClinton, A. The COVID-19 pandemic: a call to action to identify and address racial and ethnic disparities. J. Racial Ethn. Health Disparities 7, 398-402 (2020).

43. Rehm, C. D., Peñalvo, J. L., Afshin, A. \& Mozaffarian, D. Dietary intake among US adults, 1999-2012. JAMA 315, 2542-2553 (2016).

44. Power, M., Doherty, B., Pybus, K. \& Pickett, K. How COVID-19 has exposed inequalities in the UK food system: the case of UK food and poverty. Emerald Open Res. 2, 11 (2020)

45. Bhutani, S., Kahn, E., Tasali, E. \& Schoeller, D. A. Composition of two-week change in body weight under unrestricted free-living conditions. Physiol. Rep. 5, e13336 (2017)

46. Magkos, F. et al. Effects of moderate and subsequent progressive weight loss on metabolic function and adipose tissue biology in humans with obesity. Cell Metab. 23, 591-601 (2016).

47. Tackling Obesity: Empowering Adults and Children to Live Healthier Lives (UK Department of Health and Social Care, 2020).

48. Hipp, L., Bünning, M., Munnes, S. \& Sauermann, A. Problems and pitfalls of retrospective survey questions in COVID-19 studies. Surv. Res. Methods https://doi.org/10.18148/SRM/2020.V14I2.7741 (2020).

49. Nguyen, L. H. et al. Risk of COVID-19 among front-line health-care workers and the general community: a prospective cohort study. Lancet Public Health 5, e475-e483 (2020)

50. Díaz-Gutiérrez, J., Ruiz-Canela, M., Gea, A., Fernández-Montero, A. \& Martínez-González, M. Á. Association between a healthy lifestyle score and the risk of cardiovascular disease in the SUN cohort. Rev. Esp. Cardiol. 71, 1001-1009 (2018).

51. Sotos-Prieto, M. et al. A healthy lifestyle score is associated with cardiometabolic and neuroendocrine risk factors among Puerto Rican adults. J. Nutr. 145, 1531-1540 (2015)

52. Shi, D., Lee, T. \& Maydeu-Olivares, A. Understanding the model size effect on SEM fit indices. Educ. Psychol. Meas. 79, 310-334 (2019).

53. Byrne B.M. Structural Equation Modeling with AMOS: Basic Concepts, Applications and Programming (Lawrence Erlbaum, 2001).

\section{Acknowledgements}

We thank all of the participants who entered data into the app, including study volunteers enrolled in cohorts within the COPE Consortium. We thank the staff of Zoe Ltd and the Department of Twin Research at King's College London for their tireless work in contributing to the running of the study and data collection. We also thank J. Cade, Leeds University, for her feedback on the manuscript. ZOE provided in-kind support for all aspects of building, running and supporting the app and service to all users worldwide. This work was supported by the UK Department of Health via the NIHR Comprehensive Biomedical Research Centre Award (number COV-LT-0009) to Guy's and St Thomas' NHS Foundation Trust (in partnership with King's College London and King's College Hospital NHS Foundation Trust) and a grant to ZOE, and by the Wellcome Engineering and Physical Sciences Research Council Centre for Medical Engineering at King's College London (WT 203148/Z/16/Z). Funding: ZOE, Wellcome Trust (number 212904/Z/18/Z), DHSC. This research was funded in whole, or in part, by the Wellcome Trust (grant number 212904/Z/18/Z). For the purpose of open access, the author has applied a CC BY public copyright licence to any author accepted manuscript version arising from this submission.

\section{Author contributions}

S.B., T.D.S., J.W., S.O. and C.S. conceptualized the project. B.M. and M.M. curated the data. B.M., M.M., P.W.F., L.H.N., J.M., R.G., E.R.L., A.T.C., J.C.P. and M.S.G. carried out the formal analysis. S.S., C.H., E.R.L., S.B., R.G., M.M., P.W.F., A.T.C., J.M., L.M. and C.G conducted the investigation. S.B., R.G., A.T.C., P.W.F. and T.D.S. supervised the project. M.M., E.R.L., J.M., L.H.N., S.S., K.K., B.M., T.M., M.S.G., C.J.S., J.W., C.H., D.A.D., C.H.S., S.O., C.G., T.D.S., A.T.C., P.W.F., R.G. and S.B. wrote the manuscript.

\section{Competing interests}

This work was supported by Zoe Ltd. The Department of Twin Research receives grants from the Wellcome Trust (number 212904/Z/18/Z) and Medical Research Council/ British Heart Foundation Ancestry and Biological Informative Markers for Stratification of Hypertension (AIMHY; number MR/M016560/1), and support from the European Union, the Chronic Disease Research Foundation, Zoe Ltd, the NIHR Clinical Research Facility and the Biomedical Research Centre (based at Guy's and St Thomas' NHS Foundation Trust in partnership with King's College London). P.W.F., A.T.C., S.B. and E.R.L. are consultants to ZOE Ltd. T.D.S., J.W., S.S., C.H., J.C.P. and K.K. are or have been employees of ZOE Ltd. The other authors declare no competing interests. The study sponsors (ZOE Ltd; J.W.) contributed as part of the Scientific Advisory Board to the study design and collection. 


\section{Additional information}

Extended data is available for this paper at https://doi.org/10.1038/s43016-021-00398-3.

Supplementary information The online version contains supplementary material available at https://doi.org/10.1038/s43016-021-00398-3.

Correspondence and requests for materials should be addressed to Sarah E. Berry.

Peer review information Nature Food thanks Hanene Samouda and the other, anonymous, reviewer(s) for their contribution to the peer review of this work.

Reprints and permissions information is available at www.nature.com/reprints.

Publisher's note Springer Nature remains neutral with regard to jurisdictional claims in published maps and institutional affiliations.
Open Access This article is licensed under a Creative Commons Attribution 4.0 International License, which permits use, sharing, adaptation, distribution and reproduction in any medium or format, as long as you give appropriate credit to the original author(s) and the source, provide a link to the Creative Commons license, and indicate if changes were made. The images or other third party material in this article are included in the article's Creative Commons license, unless indicated otherwise in a credit line to the material. If material is not included in the article's Creative Commons license and your intended use is not permitted by statutory regulation or exceeds the permitted use, you will need to obtain permission directly from the copyright holder. To view a copy of this license, visit http://creativecommons. org/licenses/by/4.0/.

(c) The Author(s) 2021 


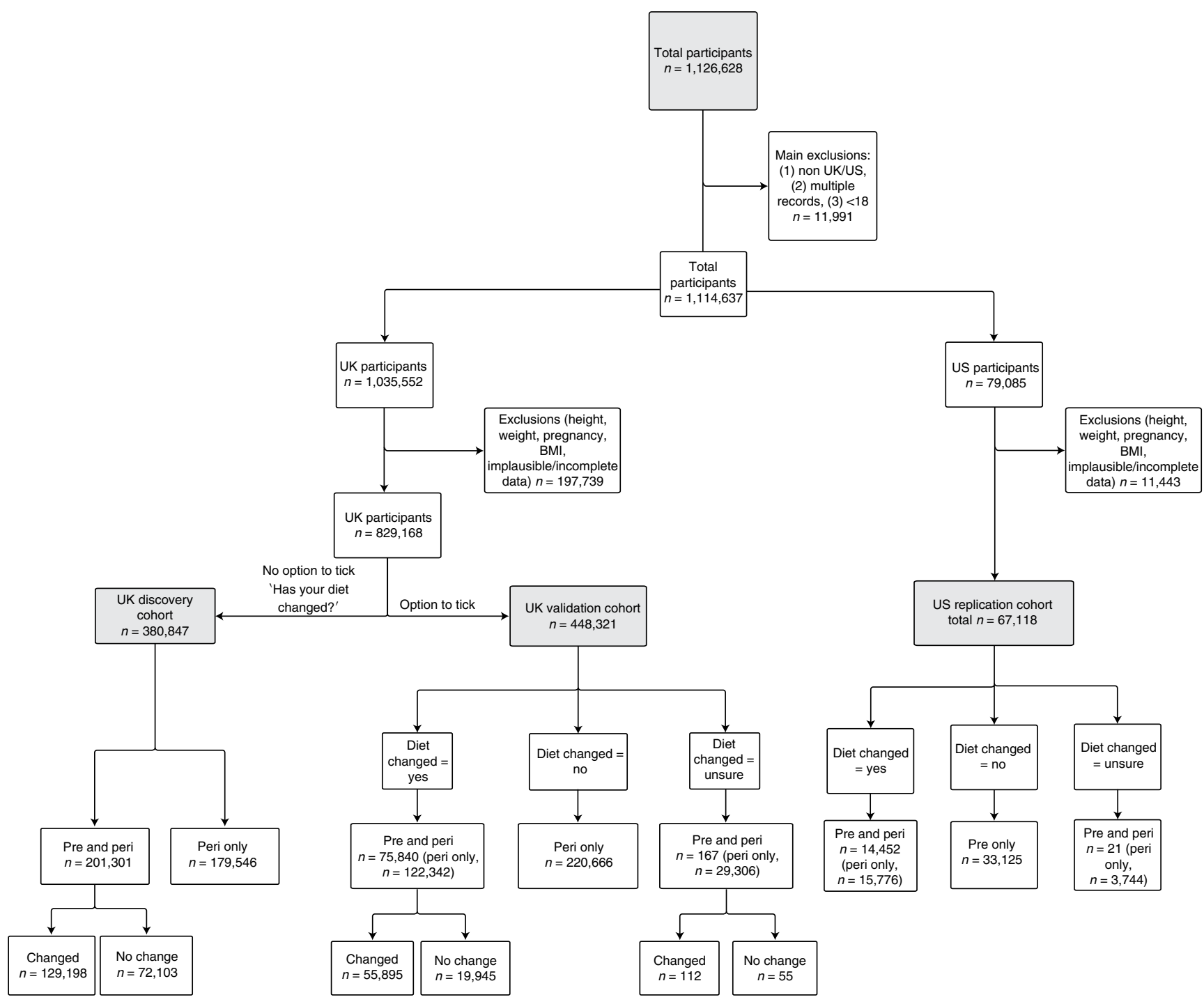

Extended Data Fig. 1 | CONSORT diagram. Figure created with BioRender.com. The data are provided in Supplementary Tables 1 and 2. 